\title{
Innovación docente para el desarrollo de la competencia transversal "Conocimiento de problemas contemporáneos" en el marco del proyecto de competencias transversales de la UPV
}

\section{Oltra-Badenes, Raúl a y Gil-Gómez, Hermenegildo ${ }^{b}$}

${ }^{a}$ Departamento de Organización de Empresas. Universitat Politècnica de València ${ }^{b}$ Departamento de Organización de Empresas. Universitat Politècnica de València

\begin{abstract}
This paper presents a teaching innovation based on the implementation of different training activities to be able to develop the competence "CT-10 Knowledge of contemporary problems" of the Transversal Competences Project of the Polytechnic University of Valencia (UPV). In order to work this competence, we have to look for training scenarios in which students have contact with their environment, always from a practical point of view, and close to the real environment in which they will find themselves in their future employment, once their studies are finished. The innovation presented is based on the use of different training activities within the subject, such as the use of Case Analysis, Projects, Report Writing and Oral Presentations, as well as the organization of talks with professionals.

In order to know the opinion of the students regarding the use of these teaching activities, a brief survey was carried out. The results obtained demonstrate the acceptance and positive evaluation by the students of this type of activities, which allow to develop the contents of the subject in a practical and applied way, while allowing the development and application of transversal competence "CT- 10 Knowledge of contemporary problems"
\end{abstract}

Keywords: Knowledge of contemporary problems, Transversal competences, Learning activities

\section{Resumen}

En este artículo se presenta una innovación docente basada en la puesta en marcha de diferentes actividades formativas para poder desarrollar la competencia "CT-10 Conocimiento de problemas contemporáneos" del Proyecto de competencias transversales de la Universitat Politècnica de València (UPV). Para trabajar esta competencia, se tienen que buscar escenarios formativos en los que los alumnos tengan contacto con su entorno, siempre desde un punto de vista práctico, y cercano al entorno real en el que se encontrarán en su futuro laboral, una vez finalizados sus estudios. La innovación que se presenta, se basa en la utilización de diferentes actividades formativas dentro de la asignatura, como es el uso de 
Innovación docente para el desarrollo de la competencia transversal "Conocimiento de problemas contemporáneos" en el marco del proyecto de competencias transversales de la UPV

\begin{abstract}
Análisis de Casos, Proyectos, Redacción de Informes y Presentaciones orales, además de la organización de charlas con profesionales.

Para conocer la opinión de los alumnos ante la utilización de estas actividades docentes, se ha realizado una breve encuesta. Los resultados obtenidos demuestran la aceptación y valoración positiva por parte del alumnado de este tipo de actividades, que permiten desarrollar los contenidos de la asignatura de una forma práctica y aplicada, al mismo tiempo que permiten el desarrollo y aplicación de la competencia transversal "CT-10 Conocimiento de problemas contemporáneos"
\end{abstract}

Palabras clave: Conocimiento de problemas contemporáneos, Competencias transversales, Actividades formativas

\title{
1. Introducción
}

El proyecto institucional de "competencias transversales" de la UPV comenzó en 2013 y el curso académico 2014/15 fue el de la experiencia piloto, en la que se realizaron diferentes actividades de prueba, con el objetivo de que el curso académico 2015/16 fuera ya el del comienzo de la implantación definitiva del proyecto.

El objetivo principal del proyecto institucional en la UPV es certificar los niveles de los alumnos en "competencias transversales", pero valorando estas a partir de actividades que las usen explícitamente. Es decir, mediante actividades dentro de las diversas asignaturas. Con esas actividades se trabajarán y desarrollarán las competencias específicas al mismo tiempo que las transversales, facilitándose así el proceso de aprendizaje de ambas competencias (UPV, 2012)

Para comprender este proyecto, es necesario definir las "competencias transversales", que podemos definir como "aquellas competencias que son claves y transferibles en relación a una amplia variedad de contextos personales, sociales, académicos y laborales a lo largo de la vida. En este sentido, constituyen una parte fundamental del perfil profesional y del perfil formativo de todas o de la mayoría de las titulaciones. Se trata de competencias que incluyen un conjunto de habilidades cognitivas y metacognitivas, conocimientos instrumentales y actitudinales de gran valor para la sociedad del conocimiento" (ICE, 2017). Se trata por tanto de un saber hacer muy complejo, por lo que es necesario concretarla en resultados de aprendizaje más específicos. Por ello, será necesario recurrir a estrategias metodológicas que propicien el cambio de roles. Ya no se puede utilizar una metodología "tradicional" basada en clases magistrales y problemas que el profesor resuelve en el aula. Incluso las prácticas de laboratorio deben cambiar su enfoque para conseguir nuevos objetivos, y lograr que los alumnos adquieran una serie de capacidades, que, por lo general, antes no se tenían en cuenta ni se evaluaban, como son la capacidad de trabajo en grupo, la capacidad de realizar exposiciones orales, etc. (Oltra-Badenes y GilGómez, 2015)

(cc) EY-NC-ND 2017, Universitat Politècnica de València 
En el proyecto de Competencias Transversales de la UPV, se han tenido en cuenta las normativas y directrices más importantes nacionales e internacionales, así como la numerosa literatura científica existente sobre las mismas, llegando a la definición del siguiente listado de competencias transversales UPV:

- CT-01. Comprensión e integración

- CT-02. Aplicación y pensamiento práctico

- CT-03. Análisis y resolución de problemas

- CT-04. Innovación, creatividad y emprendimiento

- CT-05. Diseño y proyecto

- CT-06. Trabajo en equipo y liderazgo

- CT-07. Responsabilidad ética, medioambiental y profesional

- CT-08. Comunicación efectiva

- CT-09. Pensamiento crítico

- CT-10. Conocimiento de problemas contemporáneos

- CT-11. Aprendizaje permanente

- CT-12. Planificación y gestión del tiempo

- CT-13. Instrumental específica

De entre ellas, en este artículo, se presenta una propuesta de actividades que, de forma integrada en la asignatura, van a ayudar a desarrollar y alcanzar la CT-10, Conocimiento de problemas contemporáneos.

En esta Competencia transversal, se hace referencia a la necesidad de que los estudiantes comprendan las cuestiones y valores políticos, sociales, legales y medioambientales contemporáneos, así como los mecanismos de expansión y difusión del conocimiento. Se trata de que desarrollen la capacidad de "estar al día" de los eventos actuales en su campo de conocimiento y en la sociedad en general.

Para saber si se alcanza esa competencia en concreto, se utilizan unos indicadores a modo de evidencia, para dar cuenta de que el resultado de aprendizaje ha sido alcanzado. Concretamente, el proyecto de la UPV propone utilizar los siguientes indicadores:

1. Identificar las causas que han llevado a la situación actual de un problema.

2. Identificar las consecuencias sociales, económicas, culturales y de diverso ámbito del problema en el contexto de su campo profesional.

3. Distinguir las partes que componen un problema y relacionarlas entre ellas, identificando los principales actores en sus diferentes dimensiones (económica, social, ética, tecnológica...).

4. Razonar (críticamente) las soluciones ya propuestas para un problema.

5. Proponer nuevas soluciones al problema que se ha planteado a partir de la propia experiencia y de la información disponible.

Estos indicadores, se integran en una rúbrica, que es un recurso para la evaluación y calificación del aprendizaje, de los conocimientos o del desempeño de los estudiantes en 
una actividad concreta y que establece indicadores (evidencias) y una escala de valoración para cada uno de ellos. Es decir, una rúbrica fija los criterios en los que el profesor va a centrar su atención para evaluar la actividad. Por tanto, esta rúbrica, junto con otras herramientas, se puede utilizar como elemento de evaluación. Pero evidentemente, para alcanzar las competencias, es necesario realizar acciones formativas. Dichas acciones, deben estar basadas en metodologías activas para la formación de competencias, en métodos con participación del alumno, que generan un aprendizaje más profundo, significativo y duradero (Fernández March, 2006). Además, si se utilizan metodologías activas, y cooperativas. En este caso, las que se proponen en el Proyecto UPV como más adecuadas para alcanzar la competencia CT-10, son las siguientes:

- Estudio de casos

- Dilemas éticos

- Exposiciones orales

- Foros y debates

- Juego y simulación

- Lecturas

- Preguntas

- Problemas

- Redacción de informes

- Seminario

- Visitas externas

En este artículo se presenta la aplicación de algunas de estas acciones formativas a una asignatura del Grado de Ingeniería en Organización Industrial que se imparte en la ETSII de la UPV. En concreto, de las propuestas por la UPV, las acciones formativas que se han implantado son:

- Estudio de casos

- Exposiciones orales

- Foros y debates

- Juego y simulación

- Lecturas

- Preguntas

- Redacción de informes

Además, se han añadido otras dos acciones formativas, que son:

- Proyecto

- Charlas de profesionales

Cabe destacar que, tanto la actividad formativa de Proyecto, como las Charlas de profesionales, no están definidos como actividad formativa adecuada para esta competencia. Sin embargo, como se verá más adelante, se considera que es adecuada en el contexto de la asignatura, y por ello se propone utilizarlas junto con las otras dos, para que el alumno sea capaz de alcanzar la competencia transversal.

(cc)) EY-NC-ND 2017, Universitat Politècnica de València 


\section{Objetivos}

El objetivo fundamental de la experiencia que se presenta en este trabajo, es conseguir desarrollar la competencia transversal CT-10 Conocimiento de problemas contemporáneos. Dicha competencia se desarrollará en el marco de una asignatura que se presenta a continuación.

\subsection{Contexto de la Innovación docente: La asignatura}

La asignatura en la que se realiza esta acción de innovación docente es la asignatura con código 11517, que se denomina "Sistemas Integrados de Información en Empresas Industriales" (SIIEI) y se imparte en el Grado de Ingeniería de Organización Industrial (GIOI), en la Escuela Técnica de Ingenieros Industriales (ETSII), de la UPV.

Esta asignatura se imparte en el $4^{\circ}$ curso, y en el segundo cuatrimestre, dentro de la intensificación de "Sistemas de Información y Gestión del Conocimiento". Por tanto, se puede decir que los alumnos están en la fase final del Grado, y tras finalizar la asignatura, estarán en disposición de realizar y presentar el Trabajo de Fin de Grado, e incorporarse al mercado laboral, como Graduados en Ingeniería de Organización Industrial.

Por este motivo, se considera por parte de los autores, que la CT-10 Conocimiento de problemas contemporáneos, es fundamental, debido a que, en breve, los alumnos se incorporan al mundo laboral, en el que el dominio de esta competencia es imprescindible. Seguramente, este razonamiento es compartido por la ETSII, y posiblemente sea una de las causas por las que se ha establecido, dentro del plan de estudios de la titulación, que esta asignatura sea Punto de Control de la competencia en cuestión.

\section{Desarrollo de la innovación}

Para alcanzar el objetivo anteriormente descrito, es decir, desarrollar la competencia transversal CT-10 Conocimiento de problemas contemporáneos, se han desarrollado diferentes actividades formativas, que se detallan a continuación.

\subsection{Acciones formativas llevada a cabo}

Además de las explicaciones del profesor, que en todo momento tratan de transmitir los conocimientos de forma que se puedan desarrollar las competencias transversales, es decir, explicando casos prácticos, poniendo ejemplos del entorno empresarial, utilizando preguntas, proponiendo lecturas, moderando foros y debates, etc. se han llevado a cabo algunas actividades para desarrollar la CT-10 de forma específica.

Estas son, concretamente Análisis de casos, Proyecto, Redacción de Informes, Exposición Oral y Charlas de profesionales. A continuación, se hace una explicación de cómo se han llevado a cabo cada una de estas actividades formativas. 
- Análisis de casos

Para desarrollar esta acción formativa, se presentan casos basados en situaciones reales Estos casos son facilitados a los alumnos, junto con una serie de preguntas que les hacen reflexionar sobre el mismo en relación a los objetivos de aprendizaje y competencias a alcanzar en la asignatura. Los alumnos, divididos en grupos de 4 personas, discuten la solución durante unos treinta minutos, aproximadamente. Posteriormente, se realiza una puesta en común general, en la que participa todo el grupo con el profesor. De esta forma, se consigue la participación activa y se motiva al estudiante, a la vez que se le enseña a analizar problemas. Se consigue de este modo que los alumnos puedan entrar en contacto con ideas diferentes, incluso contrarias a las propias.

De hecho, en alguno de los casos propuestos, el caso se plantea de forma que las respuestas que parecen más lógicas, o las conclusiones hacia las que parece que va enfocado el caso en función de su redacción, son erróneas, en función de algunos errores comunes que se producen en el área de conocimiento. De esta forma, se consigue que el alumno sea crítico con el caso con el que se encuentra, bien porque detecta esta situación, o bien porque no la detecta, pero en la explicación y debate del grupo, es consciente de ella.

Así se desarrolla también el pensamiento crítico del estudiante, otra de las competencias transversales de la UPV

\section{- Proyecto}

En esta actividad formativa, los alumnos deben buscar ellos mismo un Proyecto a realizar, en base a una temática indicada por el profesor. El Proyecto debe ser desarrollado en un entorno empresarial real, en el que deben buscar ellos mismos una situación problemática concreta, que requiera soluciones prácticas en relación con la asignatura y el resto de conceptos vistos en la titulación. De esta forma, en grupos de 3 alumnos, deben:

1.- Seleccionar el proyecto a ejecutar, basándose en la identificación de un problema a resolver del entorno real. 2. Planificar todos los detalles del Proyecto, definiendo y distribuyendo las tareas a realizar para llegar al objetivo. 3. Obtener y estructurar la información del entorno real (por lo general, de una empresa). 4. Desarrollar el Proyecto, proponiendo soluciones. 5. Realizar un seguimiento del proyecto. 6. Presentar el proyecto. 7. Analizar y evaluar el trabajo realizado, tanto de forma conjunta, como cada una de las aportaciones individuales.

Mediante esta metodología de proyectos, se estimula en el alumnado la iniciativa y la creatividad, pero también profundiza en la gestión del tiempo, la responsabilidad y, sobre todo, le permite formular y evaluar hipótesis, planificar, encontrar soluciones, consultar fuentes de información, redactar informes, etc.

(c) EY-NC-ND 2017, Universitat Politècnica de València 
- Redacción de informes y Exposición oral

Estas dos acciones formativas, se realizan en relación con el Proyecto. Como resultado final del mismo, los alumnos deben realizar una memoria en la que presentan de forma escrita el Proyecto, su desarrollo, planificación, resultados y conclusiones. Esto se hace en base a una guía de orientación, que se facilita a les estudiantes mediante una plantilla, con un guión que pueden seguir (pero también modificar en base a las necesidades concretas del Proyecto) para la redacción del informe final.

Finalmente, los alumnos también deben hacer una exposición oral del trabajo en clase, en la que participan todos los integrantes del grupo.

De este modo, se trabajan también las competencias transversales de Comunicación, tanto oral como escrita, que se corresponden con la CT-08 Comunicación efectiva, de las desarrolladas en el proyecto de la UPV

- Charlas de profesionales

Este tipo de acción o actividad formativa, no está recogida como tal en ninguno de los documentos relacionados con las competencias transversales de la UPV. Sin embargo, se considera una acción formativa muy interesante, en la que los alumnos se relacionan con el mundo profesional a través de las experiencias de profesionales del sector en el que, previsiblemente, trabajarán en el futuro.

En concreto, en esta asignatura, se han realizado 4 charlas de profesionales reconocidos, en relación con diferentes temas tratados en la asignatura.

\section{Resultados}

Para valorar los resultados obtenidos, se ha querido averiguar la acogida de este tipo de actividades por parte de los alumnos, y para ello se les ha realizado una pequeña encuesta que se analiza a continuación. La encuesta se ha realizado en papel, y posteriormente se ha trasladado a Google Drive, plataforma gratuita, para poder tener el registro en formato electrónico. En la encuesta a los alumnos, se les plantean cuatro cuestiones, y ellos deben valorarlas mediante una escala Likert, del 1 al 5.

Concretamente las cuestiones plantadas fueron:

Los alumnos debían valorarlas en una escala del 1 al 5, siendo:

1. Totalmente en desacuerdo

2. Más bien en desacuerdo

3. Ni de acuerdo ni en desacuerdo

4. Más bien de acuerdo

5. Totalmente de acuerdo

En el curso había 30 alumnos matriculados, y se registraron un 100\% de respuestas (30).

Las respuestas a cada una de estas preguntas se resumen en gráfica siguiente

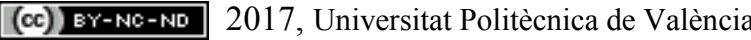




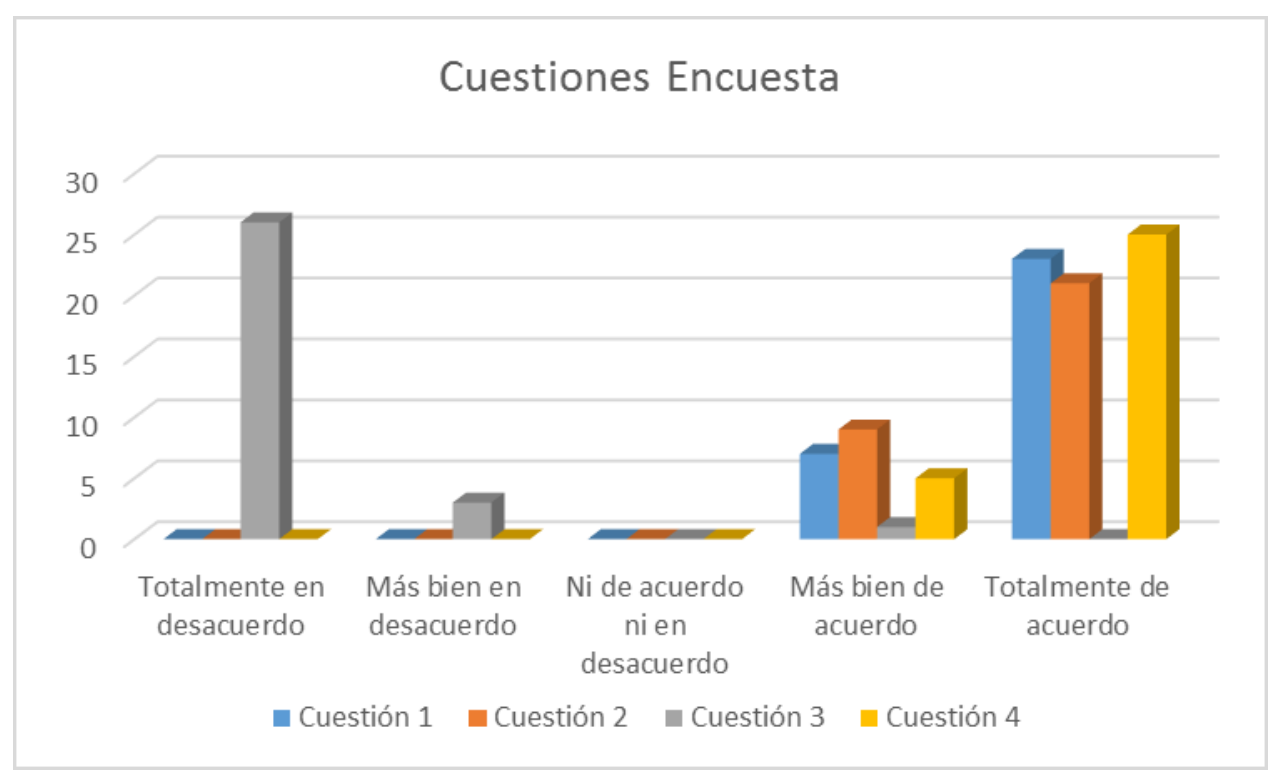

Gráfica 1. Resumen de respuestas a la encuesta realizada

En esta gráfica, se representan los valores del número de respuestas para cada pregunta.

De este modo, se observa que en la pregunta 1, ningún alumno optó por las opciones "Totalmente en desacuerdo", "Más bien en desacuerdo" y "Ni de acuerdo ni en desacuerdo", mientras que 7 alumnos declararon que estaban "Más bien de acuerdo" y 23 que estaban "Totalmente de acuerdo" con la afirmación. Es decir, el 100\% de los alumnos está de acuerdo con que las actividades desarrolladas permiten conocer mejor los problemas contemporáneos, siendo además que un $77 \%$ de los alumnos declararon estar Totalmente de acuerdo, y el 23\% restante, indicó estar Más bien de acuerdo.

Realizando el mismo análisis para las respuestas al resto de preguntas, se obtiene que el $100 \%$ de los alumnos está de acuerdo en que las actividades le ayudan a comprender la asignatura de forma práctica, sien el porcentaje de "Totalmente de acuerdo" del 70\%.

En cuanto a la pregunta en que se indica si piensan que sería mejor dedicar el tiempo a otro tipo de actividades, más tradicionales, el $97 \%$ está en desacuerdo, destacando en este caso que el $87 \%$ está Totalmente en desacuerdo. Cabe decir también que, como respuesta a esta pregunta, hay un único valor que indica estar "Más bien de acuerdo".

Por último, en cuanto a la pregunta que se hace para saber la valoración que los alumnos hacen de las actividades, el $100 \%$ indica que valora positivamente las actividades desarrolladas, siendo que el $83 \%$ está totalmente de acuerdo, y el 17\% está Más bien de acuerdo. 


\section{Conclusiones}

En este trabajo se ha presentado una acción de innovación docente en una asignatura del Grado en Ingeniería de Organización Industrial de la UPV. La innovación docente ha consistido en la implantación de diferentes actividades formativas, con el objetivo principal de desarrollar la competencia CT-10 Conocimiento de Problemas contemporáneos, en los alumnos de la asignatura al mismo tiempo que se desarrollaban los objetivos de aprendizaje de la asignatura y sus competencias específicas.

Las acciones formativas llevadas a cabo han sido: Análisis de casos, Proyecto, Redacción de Informes, Exposición oral y charlas de profesionales. Con la finalidad de obtener feedback y saber si las acciones llevadas a cabo habían sido beneficiosas para el desarrollo de la Competencia transversal, y de la asignatura en general, se desarrolló una breve encuesta, que fue pasada a los alumnos de la asignatura. Tras analizar los datos que se pueden obtener del estudio de las respuestas, se puede concluir que las acciones llevadas a cabo son de un efecto positivo evidente para el desarrollo de la actividad docente, destacando que el $100 \%$ de los alumnos encuestados (que es el 100\% de los alumnos de la asignatura) tiene una valoración positiva de las actividades desarrolladas, que el $100 \%$ declara que estas actividades les permiten conocer mejor los problemas contemporáneos, y que también el $100 \%$ declara que estas actividades les han ayudado a aprender la asignatura de forma práctica. Por tanto, se puede decir que las actividades tienen una incidencia positiva en el desarrollo de la Competencia Transversal, así como en el desarrollo de la asignatura y la motivación del alumnado, que valora de forma muy positiva este tipo de actividades formativas, prefiriéndolas a las actividades de clase tradicionales.

\section{Referencias}

FERNÁNDEZ MARCH A (2006). "Metodologías activas para la formación en competencias". Educatio Siglo XXI, 24. pp 35-56

ICE - Instituto de Ciencias de la Educación. Universitat Politècnica de València. (2015) Proyecto Competencias Transversales UPV. $\quad<$ http://www.upv.es/entidades/ICE/info/U0724624.pdf $>$ [Consulta: 27 de marzo de 2017].

OLTRA-BADENES R. y GIL-GÓMEZ, H. (2015). «Técnicas de aprendizaje cooperativo: aplicación de metodologías activas en la asignatura de Recursos Humanos en Empresas Industriales". En: 3rd International Conference on Innovation, Documentation and Teaching Technologies (INNODOCT/15). Open Innovation and Coolhunting in Education. 374-382

UPV. Universitat Politècnica de València (2012). Competencias Transversales. $<$ http://www.upv.es/contenidos/COMPTRAN/ > [Consulta: 27 de Marzo de 2017] 